\title{
Modelling considerations in finite element analyses of ankle foot orthoses
}

\author{
S. Syngellakis ${ }^{1} \&$ M. A. Arnold ${ }^{2}$ \\ ${ }^{1}$ Wessex Institute of Technology, UK \\ ${ }^{2}$ Penso Consulting Ltd, UK
}

\begin{abstract}
An asymmetric Ankle Foot Orthosis (AFO) $\mathrm{H}$ model was generated from leg surface data and a number of design parameters through a versatile graphics routine. The effect of modelling parameters, geometry, loading and constraints on the AFO mechanical behaviour was assessed through finite element analyses. Moderate thickness non-uniformity was predicted to have a small effect on AFO stiffness although the extent of this effect may also depend on the trimline position. Variation of the heel constraints was found to have a significant effect on both the maximum stress and the moment required to produce a given rotation about the ankle axis. The magnitude and distribution of stresses in critical regions, as well as the ankle moment, were all found to depend on the distribution of imposed deformation. The results of the parametric studies point towards some possible strategies for design optimisation.
\end{abstract}

Keywords: orthotics, biomechanics, design, modelling, finite elements.

\section{Introduction}

An Ankle Foot Orthosis (AFO) is a device prescribed for the management of a wide range of disorders, including cerebral palsy, hemiplegia, stroke and drop foot. Its purpose is to support and stabilise the ankle and thus improve the gait pattern. The all-plastic AFO is manufactured by vacuum forming a sheet of thermoplastic material over a plaster cast of the patient's limb; it is then trimmed to the desired shape. Computer Aided Design (CAD) and Computer Aided Manufacture (CAM) can be used in the fabrication of such, custom-made, orthotic devices [1]. Computer technology could improve the prescription service by allowing control over the variables inherent in the design of such 
devices as well as by increasing production efficiency and thereby reducing the time and cost of the manufacturing process.

The first stage of a CAM process involves taking a negative plaster cast of the patient's limb, digitising a series of profiles on the surface of this cast, as well as any anatomical landmarks and finally translating these measurements into an accurate graphical representation of the limb. The second stage is concerned with the optimum design of the device and begins with sculpting the limb geometry into an acceptable orthosis. A program performing this task should also have the capacity to modify its shape interactively altering the contours mimicking the orthotists' traditional practices. Alternatively, such "corrections" may be guided by a more sophisticated, computer-based assessment of the AFO's mechanical characteristics, which are strongly influenced by the complex trimline shape, the non-linear material properties and the thickness of an AFO [2-4].

Previous work on finite element (FE) modelling incorporated various simplifying approximations. AFOs were modelled as beams [5] but also as twodimensional solids [6]. Subsequent three-dimensional shell analyses assumed symmetry with respect to the midsagittal plane [7]. An AFO was also analysed as a three dimensional asymmetric solid [8] but this type of FE analysis is certainly less efficient than that using shell elements due to the greater number of generated degrees of freedom. In coupled lower limb - AFO models $[6,8,9]$, the possibility of slip, friction and separation was not taken into account; the loading and constraints were not defined in a realistic manner. It was also noted in previous FE work $[5,6,8]$ that loading was generally applied at the heel or toe regions as point forces, which yield artificial stress concentrations.

In this paper, the modelling focuses on custom-made AFOs whose geometry does not conform to any standard pattern. The modelling process begins with building the solid model of the leg surface representing the untrimmed AFO. A simple, manual form of silhouetting provides the coordinates of suitably selected sets of points. The solid model of the AFO itself is then generated and converted into an FE model through a rational meshing strategy. Both solid and FE modelling is performed using ANSYS, a general-purpose FE package [10]. The results of the FE analysis provide some indication of the influence of certain modelling parameters on the mechanical behaviour of an AFO. Such outcome could inform the process of reaching a final AFO design meeting desirable clinical purposes.

\section{Solid modelling}

A sketch of the leg to be fitted with an AFO is shown in Fig. 1(a). The coordinates of points along plane contours around its surface were taken off drawings. In regions of high surface curvature, such as the ankle region, a larger concentration of points was required whereas in flatter regions, as in the calf region, where the contours are smoother, the points were more spread out as indicated in Fig. 1(a). The origin of the adopted Cartesian coordinate system was placed at the expected location of the ankle joint; the $x$ axis pointed anteriorly, the $y$ axis superiorly and the $z$ axis rightward. 
Once the coordinate data were imported into ANSYS, the contours of 17 cross-sections were generated through a spline fit to the series of points for each cross-section. A single, continuous area was then formed by 'skinning' a surface through the set of these contours, which effectively act as ribs over which the surface is stretched. As shown in Fig. 1(b), the built model represented the leg surface with reasonable accuracy, despite the relatively small number of imported points.

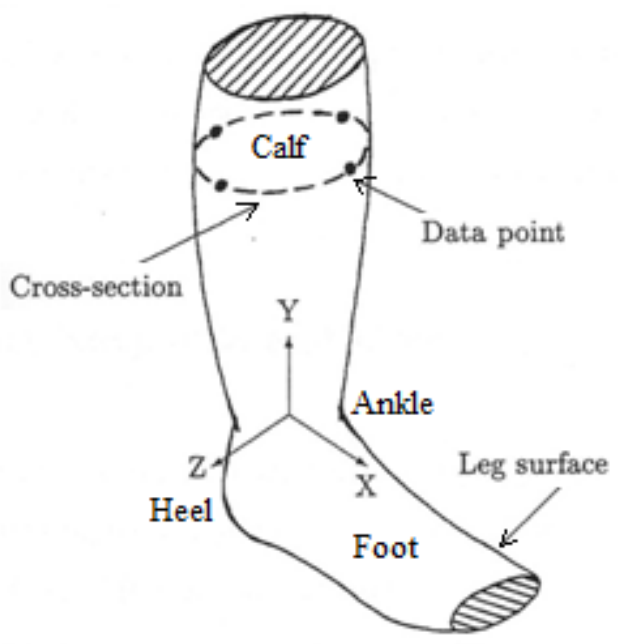

(a)

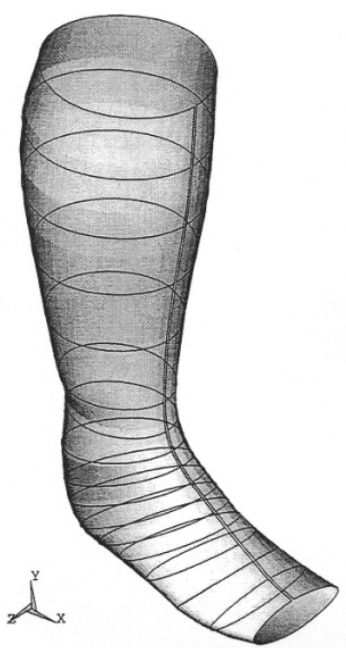

(b)

Figure 1: (a) Sketch and (b) solid model of the leg to be fitted with an AFO.

The trimming process generating a custom-made AFO from the vacuumformed thermoplastic sheet was duplicated in ANSYS by firstly defining the required trimlines for a particular design and then sculpting the solid model of the leg surface. Since its thickness is relatively small compared to its other dimensions, an AFO can be represented by areas each with a specified thickness.

For design optimisation purposes, it was necessary to define the trimlines through a set of geometric parameters. It was also convenient to keep the number of these parameters as small as possible. In actual AFOs, there are small differences between the trimlines on the medial and those on the lateral edges of an AFO when projected on the sagittal plane. In order to simplify the modelling process, it was assumed that these projections were identical. Thus, a set of physical dimensions was defined from which the single required trimline projection was generated as shown in Fig. 2. These dimensions were the height of the proximal trimline $h$, the location of the distal trimline $d$, the length and overlap of the calfband trimline $c$ and $p$, respectively, the radius of the ankle trimline and calf trimline $\operatorname{arcs} R$ and finally the ankle joint vertical clearance $a$. With this method of trimline definition, it would be easy to alter the AFO design by simply changing the values of the trimline parameters. 


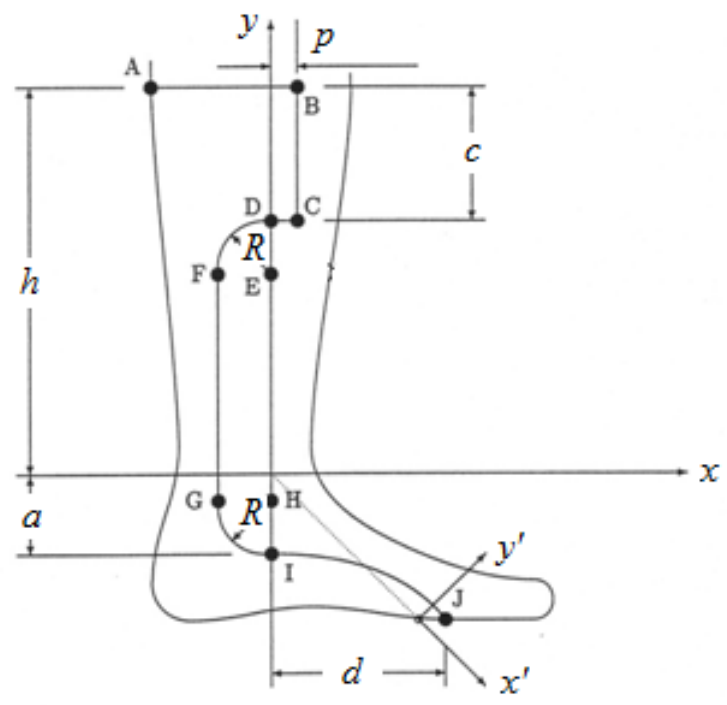

Figure 2: $\quad$ Parameters defining AFO trimlines.

The co-ordinates of the points necessary for constructing the trimlines projection, labelled from ' $\mathrm{A}$ ' to ' $\mathrm{J}$ ' in Fig. 2, were defined on a plane parallel to, and at a distance from, the $x-y$ plane in terms of these six parameters. This contour was projected back onto the leg area using the area drag operation, which generated areas by sweeping the specified pattern of lines along a drag path defined as a straight line parallel to the $z$ axis as shown in Fig. 3(a). The leg area was thus cut at the true trimlines to generate the solid model of the AFO shown in Fig. 3(b).

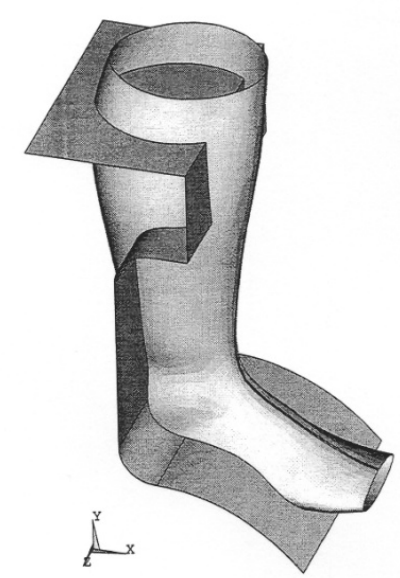

(a)

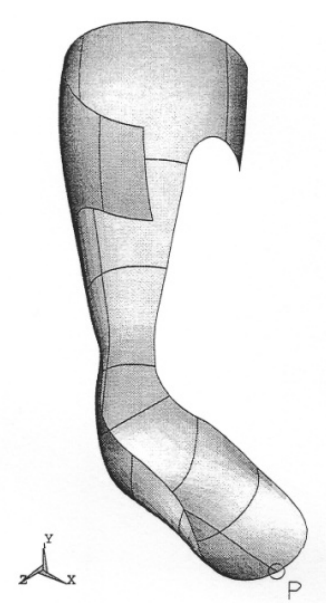

(b)

Figure 3: (a) Leg sculpting operation and (b) the resulting AFO model. 


\section{Finite element model}

Due to its thinness, a plastic AFO can be accurately analysed using shell theory. For this purpose, the 8-node (SHELL93) ANSYS shell element was adopted since it is suitable for modelling the doubly curved surface of a plastic AFO. The thickness of such an element should ideally be less than one-fifth of the radius of curvature. It should be born in mind while interpreting the results that the areas to be meshed are generated from data points corresponding to the inner surface of the AFO manufactured to fit a leg.

As mentioned above, the initial AFO solid model comprised a single solid area. In order to facilitate mesh control and generate meshes of better quality, the AFO area was divided into a number of separate but connected areas as shown in Fig. 3(b). The typical FE model shown in Fig. 4 was obtained by "free" meshing, that is, without any restriction on element shapes; as a result, the AFO areas were meshed with a mixture of mostly quadrilateral but also triangular elements. "Mapped" meshing, generating only quadrilateral elements, is expected to perform better but the conditions for applying it in an efficient and versatile manner could not be satisfied in the present case.

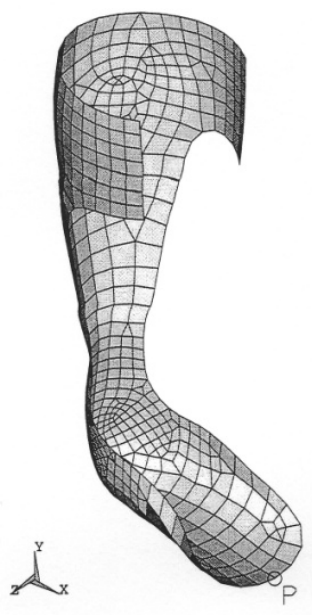

Figure 4: A finite element model of the analysed AFO.

The initial mesh shown in Fig. 4 was obtained using default element size specifications which results in a more or less uniform mesh; this may not be satisfactory since a finer mesh will be required in regions of high stress gradients. In such cases, it would be necessary, for instance, to specify the edge length of elements nearest a particular corner point or a certain number of divisions be forced on lines. 


\section{Input}

The thickness of an AFO varies over its volume due to its non-developable surface; thus, when vacuum forming a sheet of plastic material over the mould, it stretches in a non-uniform manner. The minimum thickness of a custom-made AFO, located at the heel region, was $54 \%$ of the $2-\mathrm{mm}$ maximum located at the posterior calf region. The average thickness around the ankle trimline was found approximately equal to $80 \%$ of the maximum, which agreed with similar measurements by other investigators [11]. The AFO model was analysed with a constant thickness of $2 \mathrm{~mm}$ throughout. The effect of thickness variation on the FE results was however investigated. For this purpose, individual elements were grouped into regions of constant thickness. These regions corresponded to the areas into which the solid model had already been divided as shown in Fig. 3(b). Thickness values were assigned to the various areas such that their ratios to the maximum thickness were the same as the respective ratios in the custom-made AFO.

All parametric studies were performed with the trimline dimensions of a custom-made AFO. In order to study the effect of changing trimline position on AFO stiffness, the trimline dimensions of the model were also matched to those of a pre-fabricated AFO. Both sets of values for the trimline parameters defined in Fig. 2 are given in Table 1.

Table 1: $\quad$ AFO trimline dimensions.

\begin{tabular}{|c|c|c|}
\hline \multirow{2}{*}{$\begin{array}{c}\text { Trimline } \\
\text { parameter } \\
\text { (Figure 2) }\end{array}$} & \multicolumn{2}{|c|}{ Dimensions (mm) } \\
\cline { 2 - 3 } & $\begin{array}{c}\text { "Custom- } \\
\text { made" }\end{array}$ & "Pre-fab" \\
\hline \hline$p$ & 260 & 250 \\
\hline$d$ & 110 & 115 \\
\hline$c$ & 80 & 75 \\
\hline$p$ & 30 & 20 \\
\hline$R$ & 30 & 35 \\
\hline$a$ & 30 & 53 \\
\hline
\end{tabular}

The AFO material may be orthotropic as orientation is induced during the manufacture of the thermoplastic sheet in the direction of draw [12]. Above the glass transition temperature, the relationship between stress and strain is nonlinear [13]. The visco-elasticity of the material may also affect the response of an AFO especially if subjected to repeated, cyclic loading. For the present modelling objectives, it was thought sufficient to consider the material homogeneous, isotropic and linearly elastic. Although not an exact description of polymers, linear elasticity can predict their low-strain, short-term behaviour with acceptable accuracy. The Young's modulus $E$ and Poisson's ratio $v$ were taken equal to $1000 \mathrm{MPa}$ and 0.35 respectively. The adopted value for $E$ is consistent with literature data for Copolymer Polypropylene. No exact data for $v$ of this 
particular polymer were available but, for most plastics, $v=0.35-0.45$, and a small variation in the value of $v$ does not have any significant effect on the results.

Partial constraints were applied to all the nodes of the two posterior areas in the proximal calf region to simulate the radial constraint due to the calf strap. This was achieved by rotating the nodal co-ordinate system for each node about its $y^{\prime}$-axis such that its $x^{\prime}$-axis intersected the global $y$-axis. The constraints were then applied in the nodal $x^{\prime}$-directions of each of these nodes, while their remaining degrees of freedom were left unconstrained. The AFO was therefore free to move up and down the calf, or undergo internal and external rotations. Constraints were also applied to all nodes in the four areas of the heel region to simulate the constraint provided by the shoe and foot. The nodal co-ordinate system of each node was first rotated such that its $x^{\prime}$-axis passed through the global origin. Each of these nodes was then constrained in this direction, coincident to the radial direction of a spherical co-ordinate system, while remaining free to displace in all other directions.

The model was deformed using various strategies with the objective in each case to induce $5^{\circ}$ plantar flexion of the foot section about the ankle joint axis, assumed to be the $z$-axis of the global co-ordinate system. It was not necessary to repeat the analyses for dorsiflexion because, with a linear analysis and such small imposed deformation, the results would be approximately the same in magnitude, although opposite in sign. Displacements on the distal foot region compatible with $5^{\circ}$ rotation were imposed. Such a displacement was applied at (a) a single node located at the centre of the distal trimline (point ' $\mathrm{P}$ ' in Fig. 3(b)); (b) all nodes located on the interface of the two distal areas of the foot region; (c) the nodes on a patch in the centre of the same two areas; (d) all nodes on the same two distal areas. Referring to the rotated nodal co-ordinate system, $x^{\prime} y^{\prime} z^{\prime}$, shown in Figure 2, a displacement was applied in the nodal $y^{\prime}$-direction of magnitude $r \theta$, where $r$ is the perpendicular distance from this node to the global $z$-axis and $\theta$ is the angle of rotation.

The reactions obtained from this kinematic loading had a highly irregular distribution, not reflecting the rather smooth interaction expected between AFO and foot. For this reason, the analysis was also performed with a uniformly distributed pressure, applied over the same area as the kinematic loadings. The originally specified constraints at the foot/heel region were found to provide insufficient resistance against a rigid body rotation of the AFO caused by the offcentre resultant of the pressure acting on the asymmetric foot region. Therefore, an additional constraint was imposed in the global $Z$-direction at a single node in the heel to eliminate this.

\section{Results and discussion}

\subsection{Influence of mesh density}

The analysis of a continuous solid by the finite element method leads to the so called discretisation error. An objective estimate of this error is possible within 
ANSYS based on the $Z^{2}$ error estimator $\eta$ [14]. This quantity represents the error associated with a particular mesh discretisation, and is not an absolute measure of error [10]. A series of analyses were performed under uniform pressure applied on the distal foot regions and the $\eta$ distributions obtained as contour plots. Starting from the initial mesh, the element sizes were manually refined iteratively at high error regions, around the ankle trimlines where the high stress gradients were also located. Also, the mesh was made coarser where the stress gradients were low. Thus, a sequence of 5 meshes, A-E, was generated as described in Table 2.

Table 2: $\quad$ Effect of mesh refinement.

\begin{tabular}{|c|c|c|c|c|}
\hline Mesh & $\begin{array}{c}\text { Number } \\
\text { of Nodes }\end{array}$ & $\begin{array}{c}\text { Number of } \\
\text { Elements }\end{array}$ & $\begin{array}{c}\text { Energy Error } \\
\eta(\%)\end{array}$ & $\begin{array}{c}\text { Maximum } \sigma_{e} \\
(\mathrm{MPa})\end{array}$ \\
\hline \hline $\mathrm{A}$ & 1848 & 597 & 22.9 & 24.9 \\
\hline $\mathrm{B}$ & 1708 & 549 & 20.4 & 25.7 \\
\hline $\mathrm{C}$ & 2229 & 724 & 15.9 & 26.3 \\
\hline $\mathrm{D}$ & 3691 & 1224 & 12.6 & 26.8 \\
\hline $\mathrm{E}$ & 5028 & 1667 & 10.6 & 27.2 \\
\hline
\end{tabular}

The plantar flexion angle was found to be insensitive to mesh density over the applied FE meshes but, as indicated in Table 2, the maximum von Mises stress $\sigma_{e}$ appeared to be converging to a gradually increasing value. This is because local mesh refinement enhances accuracy in regions of stress concentration. The percent energy error also decreased considerably, indicating a more reliable solution. By comparing the results for mesh B with those for mesh A, it can be noted that although the number of nodes, elements and hence solution time had reduced, the accuracy of the maximum equivalent stress had increased and the error decreased. Therefore, by controlling the density of the mesh at high and low stress gradient regions, it is possible to produce a more efficient mesh than that obtainable using only global specification.

\subsection{Influence of trimline and non-uniform thickness}

As shown in Table 1, the values of $R$ and $a$ in the "pre-fab" are greater by $17 \%$ and $77 \%$, respectively, than those in the "custom-made" trimline implying that the former define a substantially weaker AFO. The analysis of both AFOs, performed with the same uniform thickness and under the same pressure on the distal foot regions, confirmed this expectation predicting $182 \%$ and $164 \%$ increases in maximum displacement and ankle rotation, respectively. The maximum predicted $\sigma_{e}$ did not change appreciably but the direct stress changed in the critical ankle area from being combined flexural-membrane to almost purely flexural. This means that it is less likely to cause buckling.

The effect of non-uniform thickness was assessed by analysing the AFO with mesh B of Table 2 under uniform pressure on the distal foot region; comparison of the results obtained with the thickness variation described in Section 4 to 
those with the uniform 2-mm thickness, showed that a partial reduction in thickness has a rather small effect on the model's stiffness and stress levels. A uniform reduction in thickness to its minimum of $1.4 \mathrm{~mm}$ brought about a more dramatic increase of predicted rotation by about $80 \%$ accompanied by similar increases in stress magnitudes.

A second numerical test on the effect of thickness variation was based on the data from the pre-fabricated AFO whose thickness was found to vary more severely than that of the custom-made AFO although the locations of the extreme values were the same. The flexibility and maximum $\sigma_{e}$ of the model with variable thickness were found, respectively, by about $26 \%$ and $34 \%$ lower than those predicted with uniform thickness. This indicates a greater influence of thickness variation in a more flexible AFO.

\subsection{Effect of constraints and validation}

Analyses were performed with the originally specified constraints and with some of the constraints at the heel region relieved so that only those nodes on the cup of the heel were constrained. Kinematic loading (c), as described in Section 4 was applied. The results are given in the top two rows of Table 3 where $\theta_{f}$ is the actual angle of rotation of the node at the middle of the distal trimline (point ' $\mathrm{P}$ '), $\theta_{h}$ the rotation of a node at the heel region, $F$ the resultant reaction force over the displaced nodes and $M$ the total moment about the ankle joint axis of the reaction forces at these nodes. The most noticeable effect of reduced constraint on results was a corresponding $38 \%$ reduction in the maximum $\sigma_{e}$ at the ankle trimline and the re-location of that maximum from the medial to the lateral edge. The reason for this was that the stresses were more widely distributed over this region. The ankle moment was found to have decreased by $26 \%$, indicating the importance of the mode by which the heel is constrained.

Table 3: $\quad$ Effect of varying heel constraints.

\begin{tabular}{|c|c|c|c|c|c|}
\hline $\begin{array}{c}\text { Heel } \\
\text { Constraint }\end{array}$ & $\begin{array}{c}\text { Foot } \\
\text { Rotation } \\
\theta_{f} \\
\text { (degrees) }\end{array}$ & $\begin{array}{c}\text { Heel } \\
\text { Rotation } \\
\theta_{h} \\
\text { (degrees) }\end{array}$ & $\begin{array}{c}\text { Resultant } \\
\text { Foot } \\
\text { Force } F \\
(\mathrm{~N})\end{array}$ & $\begin{array}{c}\text { Ankle } \\
\text { Moment } \\
M \\
(\mathrm{Nm})\end{array}$ & $\begin{array}{c}\text { Maximum } \\
\sigma_{e} \\
(\mathrm{MPa})\end{array}$ \\
\hline \hline Full & -5.0 & -4.8 & -50.4 & -4.7 & 34.5 \\
\hline Reduced & -5.0 & -4.8 & -37.8 & -3.5 & 21.5 \\
\hline None & -4.9 & -2.5 & -6.2 & -1.0 & 9.5 \\
\hline
\end{tabular}

Another simplified analysis was performed adopting the support conditions of previously reported analyses [5, 7], that is, all heel constraints were removed and the model was rendered vertically stable by additionally constraining the calf at the nodes along the proximal edge in the global $y$-direction. The results, appearing in the 3rd row of Table 3, showed that, as the heel region was not forced to rotate about the ankle joint axis, the maximum $\sigma_{e}$ was $28 \%$ of that obtained with the heel constrained. As above, this was also located on the lateral 
edge. The main reason for this was the more widely distributed deformations in the calf region, as the whole heel region translated posteriorly rather than rotating. The total reaction and moment were also found to be $12 \%$ and $21 \%$ of the respective values obtained previously. This result showed that adopting possibly unrealistic constraints could lead to an underestimate of stress in critical regions of the AFO as well as an unreliable assessment of the ankle moment.

The results from that last analysis served as a means of validating the developed FE model. For this purpose, a section at mid-height along the shank was considered as a thin-walled beam section in the shape of a circular arc. Considering the AFO as a cantilever beam subjected to an end-load, it was possible to apply simple beam theory and find estimates of the extreme values of stresses on that section. The good agreement of those estimates with the respective FE predictions provided a degree of confidence in the validity of the model which was reinforced by the consistency and rational behaviour of the other results.

\subsection{Comparison of imposed rotation strategies}

The key results obtained from the four analyses corresponding to loading patterns (a)-(d), described in Section 4, are listed in Table 4. It is noted that the $\theta_{f}=5^{\circ}$ rotation of the foot region about the ankle axis is achieved every time, although a slight variation was present due to approximations made in the calculation of the imposed displacement.

Table 4: $\quad$ Summary of results from imposed displacement.

\begin{tabular}{|c|c|c|c|c|c|}
\hline $\begin{array}{c}\text { Displaced } \\
\text { Distal Foot } \\
\text { Entity }\end{array}$ & $\begin{array}{c}\text { Foot } \\
\text { Rotation } \\
\theta_{f} \\
\text { (degrees) }\end{array}$ & $\begin{array}{c}\text { Heel } \\
\text { Rotation } \\
\theta_{h} \\
\text { (degrees) }\end{array}$ & $\begin{array}{c}\text { Resultant } \\
\text { Foot } \\
\text { Force } F \\
(\mathrm{~N})\end{array}$ & $\begin{array}{c}\text { Ankle } \\
\text { Moment } \\
M \\
(\mathrm{Nm})\end{array}$ & $\begin{array}{c}\text { Max } \\
\sigma_{e} \\
(\mathrm{MPa})\end{array}$ \\
\hline \hline (a) Point & -4.9 & -3.4 & -23.7 & -3.3 & 24.5 \\
\hline (b) Line & -5.0 & -4.6 & -46.6 & -4.5 & 33.5 \\
\hline (c) Sub-area & -5.0 & -4.8 & -50.4 & -4.7 & 34.5 \\
\hline (d) Area & -5.0 & -4.9 & -61.4 & -4.8 & 34.6 \\
\hline
\end{tabular}

Plots of $\sigma_{e}$ obtained under all patterns of kinematic loading revealed that its maximum occurred on the inner surface of the AFO in a region of stress concentration close to the medial ankle part of each trimline. An artificial stress concentration was detected around the node where the displacement was imposed; this was reduced when displacement was imposed along a line, while the maximum $\sigma_{e}$ at the ankle trimline was increased by $37 \%$ as indicated in Table 4. It was thus confirmed that, when a displacement is applied at a single node, the FE stress results might be unreliable in the immediate vicinity of as well as far from this node. It is noted that distributing the imposed displacements over a greater region caused the heel rotation and the ankle moment to increase by $35 \%$ and $36 \%$, respectively. 
The results under loadings (c) and (d) indicate that the effects of further distributing the imposed displacements were less significant. The predicted maximum $\sigma_{e}$ increased by only $3 \%$ under loading (d) compared to that predicted under loading (b), while the rotation at the heel and the ankle moment increased by $7 \%$. In contrast, the total reaction force increased by $32 \%$, but this was only due to a large proportion of this reaction being distributed over the nodes nearest the heel region, where the moment arm was smaller.

\subsection{Comparison between pressure and kinematic loading}

For a meaningful comparison between the two types of loading, the AFO was analysed again under imposed deformation, but with the additional heel constraint in the $Z$-direction, and the total reaction force obtained was divided by the total distal foot area to yield the magnitude of the uniform pressure. The ankle moment from the analysis under pressure was lower due to some of the pressure being applied on the lateral wall of the foot region. Thus, the maximum $\sigma_{e}$ was found 5\% lower than that given in the last row of Table 4, although its location was the same. It is worth noting that, although the rotation at the distal trimline was the same for both loading types, the rotation at the heel was smaller by $10 \%$ under uniform pressure probably due to the flexibility of the foot region.

\section{Conclusions}

It has been shown in this paper that it is possible to generate a fairly realistic geometrical representation of an AFO, designed to fit a particular patient, using the CAD facilities of a general-purpose FE package. An initial assessment of the stiffness characteristics of the device can be achieved through a linear elastic analysis. Work focused on the various aspects of simulation, which may influence the accuracy of the FE calculations. Parametric studies were performed concerned with the modelling of geometry, support and applied loading.

Variable thickness is not necessarily an adverse factor, therefore lighter AFO designs can be achieved without severely affecting the required stiffness. Stiffness can be controlled by varying the thickness or the trimline position in critical areas, which are located mainly around the ankle region. The effect of realistic loading and constraint conditions is difficult to assess but, in this paper, extreme, and some intermediate, cases, corresponding to various degrees of imposed rigidity, were considered. It was found that the way loading and constraint combinations are applied can have a significant effect on predicted stiffness.

Having established the validity of certain essential features of the FE model, the latter can be used with greater confidence in AFO design. The proposed analysis can be easily adjusted to be performed under modified data and thus quantify the sensitivity of the design to such variations. 


\section{References}

[1] Boone, D.A., Harlan, J.S. and Burgess, E.M., Automated fabrication of mobility aids: review of the AFMA process and VA/Seattle ShapeMaker software design. Journal of Rehabilitation Research and Development, 31(1), pp. 42-49, 1994.

[2] Condie, D.N. and Meadows, C.B., Some Biomechanical Considerations in Design of Ankle-Foot Orthoses. Orthotics and Prosthetics, 31(3), pp. 4552, 1977.

[3] Lehmann, J.F., Esselman, P.C., Ko, M.J., Smith, J.C., Delateur, B.J. and Dralle, A.J., Plastic Ankle-Foot Orthoses - Evaluation of Function. Archives of Physical Medicine and Rehabilitation, 64(9), pp. 402-407, 1983.

[4] Sumiya, T., Suzuki, Y. and Kasahara, T., Stiffness control in posterior-type plastic ankle-foot orthoses: Effect of ankle trimline .2. Orthosis characteristics and orthosis/patient matching. Prosthetics and Orthotics International, 20(2), pp. 132-137, 1996.

[5] Leone, D.J., A Structural Model for Molded Thermoplastic Ankle-Foot Orthoses. Journal of Biomechanical Engineering-Transactions of the Asme, 109(4), pp. 305-310, 1987.

[6] Lam, P.C., Reddy, N.P. and Downing, M., Effects of heel and toe forces on ankle-foot orthoses design using finite element analysis. ASME Design Engineering Division (Publication), vol. 1, pp. 49-52, 1986.

[7] Leone, D., Diemente, S., Gustave, S. and Lopez-Isa, M., Structural analysis of solid ankle-foot orthoses. Proc. of the 14th Annual Northeast Bioengineering Conf., Durham, NH, pp. 26-28, 1988.

[8] Chu, T.M., Reddy, N.P. and Padovan, J., 3-Dimensional Finite-Element Stress-Analysis of the Polypropylene, Ankle-Foot Orthosis - Static Analysis. Medical Engineering and Physics, 17(5), pp. 372-379, 1995.

[9] Lam, P.C., Reddy, N.P. and Downing, M., Dynamic responses of anklefoot orthoses. ASME Bioengineering Division (Publication), vol. 2, pp. 130$131,1986$.

[10] ANSYS, Inc., ANSYS Academic Research, Canonsburg, PA 15317, Www.ansys.com

[11] Golay, W., Lunsford, T., Lunsford, B.R. and Greenfield, J., The Effect of Malleolar Prominence on Polypropylene Afo Rigidity and Buckling. Journal of Prosthetics and Orthotics, 1(4), pp. 231-241, 1989.

[12] Birley, A.W. and Scott, M.J., Plastic Materials: Properties and Applications, Leonard Hill: Glasgow, pp. 1982.

[13] Williams, J.G., Stress Analysis of Polymers, Longman: London, pp. 1973.

[14] Cook, R.D., Finite Element Modelling for Stress Analysis, John Wiley and Sons: Chichester, pp. 1995. 